Short Report

\title{
Cytokine Induced Killer Cells as Promising Immunotherapy for Solid Tumors
}

\section{Dario Sangiolo ${ }^{凶}$}

Laboratory of Cell Therapy of Cancer, Institute for Cancer Research and Treatment, Candiolo (Torino), Italy

$\triangle$ Corresponding author: dario.sangiolo@ircc.it

( ) Ivyspring International Publisher. This is an open-access article distributed under the terms of the Creative Commons License (http://creativecommons.org/ licenses/by-nc-nd/3.0/). Reproduction is permitted for personal, noncommercial use, provided that the article is in whole, unmodified, and properly cited.

Received: 2011.05.16; Accepted: 2011.06.08; Published: 2011.06.10

\begin{abstract}
Cytokine-induced killer (CIK) cells are a heterogeneous subset of ex-vivo expanded T lymphocytes which present a mixed T-NK phenotype and are endowed with a MHC-unrestricted antitumor activity. The main functional properties of CIK cells may address some of the main limitations that are currently preventing the successful clinical translation of adoptive immunotherapy strategies. Clinically adequate quantities of immune effectors, sufficient for multiple adoptive infusions, may be obtained based on their relatively easy and inexpensive ex-vivo expansion starting from peripheral blood mononuclear cells. The MHC-unrestricted tumor-killing is mainly based on the interaction between NKG2D molecules on CIK cells and MIC A/B or ULBPs molecules on tumor cells; it has been proved effective against several solid and hematological malignancies and does not require any HLA-restriction increasing the number of patients that might potentially benefit from such approach. Finally, CIK cells present a reduced alloreactivity across HLA-barriers with important clinical implications for their potential use as alternative to conventional Donor Lymphocyte Infusions after allogeneic hemopoietic cell transplant with a reduced risk of GVHD. In the present report we review the main functional characteristics of CIK cells discussing recent findings and future perspectives to improve their antitumor activity and potential clinical applications.
\end{abstract}

Key words: CIK cells; adoptive immunotherapy; solid tumors.

\section{Introduction}

Adoptive immunotherapy holds great promises in the scenario of potential new approaches for the treatment of solid tumors refractory to conventional therapies. Crucial issues for all adoptive immunotherapy strategies include the obtainment of sufficient numbers of immune effectors, recognition of tumor targets and possible restriction to specific HLA-haplotypes. Cytokine-Induced Killer (CIK) cells are a heterogeneous subset of ex-vivo expanded $\mathrm{T}$ lymphocytes whose biological features make them appealing for adoptive immunotherapy, addressing some limitations associated with other strategies tar- geting specific Tumor-associated antigens (TAA).The main functional properties that favorably characterize CIK cells are: 1) Ex-vivo expansion 2) Reduced alloreactivity; 3) MHC-unrestricted tumor-killing.

\section{Ex-vivo expansion and phenotype of CIK cells}

An important limitation preventing the successful clinical translation of several adoptive immunotherapy strategies is the obtainment of sufficient numbers of anti-tumor immune effectors and their in-vivo persistence after infusion into the patients. An 
important positive characteristic of CIK cells is their easy, and relatively inexpensive, ex-vivo expansion [1, 2]. CIK cell precursors are CD3+ $\mathrm{T}$ lymphocytes, mainly with a naïve, CD4CD8 double negative (CD4-CD8-) phenotype [3]. They can be classically expanded starting from peripheral blood mononuclear cells (PBMC) but may also be generated from bone marrow or umbilical cord blood precursors [3, 4]. The standard culture conditions require three to four weeks with the timed addition of IFN- $\gamma, \mathrm{Ab}$-anti CD3 and IL2 [3]. IFN- $\gamma$ is only added on day 0 , its main activity is to activate the monocytes, present in the initial bulk culture, which provide both contact-dependent (CD58/LFA-3) and soluble (IL12) crucial signals that favor the acquisition of a final Th1 phenotype and cytotoxic power of CIK cells [5-7]. The $\mathrm{Ab}$ anti-CD3 provides mitogenic signals to $\mathrm{T}$ lymphocytes subsequently sustained by IL2 that drives the expansion $[8,9]$. Some groups have reported the addition of IL7 as beneficial to increase the cytotoxic potential of CIK cells [10]. After 3-4 weeks of culture the expansion rate is reported to be variable from few to more than 1000 fold [2, 11-13]. Such levels of expansion, also considering the availability of CD3+ starting precursors, are extremely favorable for subsequent clinical applications. The reason of the reported variability in the range of expansion rates is not clear, additional experimental strategies are currently under investigation to further ameliorate such numbers, especially for those patients who may be in the lower range ("poor expanders") and may less benefit from such approach. Alternative strategies are based on additional soluble factors added to culture conditions (Thymoglobulin, IL1, IL7) [14] [10], addition of transient allogeneic stimulation or depletion of $\mathrm{T}$ regulatory cells during the expansion culture [15]. Our group recently reported that a transient allogeneic stimulation with allogeneic irradiated PBMC may provide an important additional expansion boost to CIK cells without affecting their antitumor activity of safety profile [16]. Standard CIKs' culture conditions have been successfully validated under Good Manufacturers Practice (GMP) conditions [4] and have allowed recent applications into clinical trials. At the end of the expansion there is a heterogeneous population of $\mathrm{CD} 3+\mathrm{T}$ lymphocytes, with two main subsets, respectively positive $(\mathrm{CD} 3+\mathrm{CD} 56+)$ and negative (CD3+CD56-) for the simultaneous expression of CD56 membrane molecule. The CD3+CD56+ fraction is considered the main responsible for the MHC-unrestricted antitumor activity [3, 13]. Mature CIK cells are mainly CD8+ but CD4+CD8- cells can also be present in the bulk culture, less frequent are subsets with a double positive $(\mathrm{CD} 4+\mathrm{CD} 8+)$ or double negative (CD4-CD8-) phenotype. The terminally differentiated late effector phenotype (CD45RO+; CD27low; CD28low; CD62L-; CCR7-) is the more represented among $\mathrm{CD} 3+\mathrm{CD} 56+\mathrm{CIK}$ cells while the CD3+CD56- counterpart exhibits more earlier memory characteristics [17].

\section{Alloreactivity of CIK Cells}

A second clinically relevant property of CIK cells is their reduced alloreactive potential across MHC barriers that may result in a reduced risk for GVHD if adoptively infused after allogeneic Hemopoietic Cell Transplant (HCT) [13]. Early preclinical data, obtained from murine models of HCT, demonstrated that the adoptive infusion of allogeneic CIK cells across MHC-barriers was associated with a lower risk of acute GVHD compared to conventional T cells. The main biological explanation for this beneficial effect is likely linked to the abundant production of IFN- $\gamma$, by expanded CIK cells, and its known protective action against GVHD [18, 19]. Compared to conventional T lymphocytes, allogeneic CIK cells displayed a significant lower acquisition of homing molecules, required for the entry of inflamed and GVHD target organs (a4 37, CCR9, E-selectin, CXCR3 and CCR5) and a higher susceptibility to apoptosis [20].

Even if murine studies confirmed a reduced GVHD potential, initial clinical trials infusing donor CIK cells after HLA identical HCT, reported a $36 \%$ of GVHD incidence [21]. These events were of low grade but sufficient however to raise concerns about a residual alloreactivity of CIK cells that might become clinically relevant when challenged across major HLA-barriers. Preclinical studies confirmed that human CIK cells retain a behavior similar to conventional $\mathrm{T}$ lymphocytes, being able of intense proliferation if stimulated across major HLA-barriers. Such alloreactive-driven proliferation is however mainly restricted to the CD3+CD56- subset [13]. The first important implication is that a depletion of the "alloreactive" (CD3+CD56-) CIK fraction from the expanded bulk population may further reduce their alloreactive and consequent GVHD potential. This approach may be of particular relevance in peculiar HCT settings with augmented degree of HLA-mismatch between donor and recipient (i.e. Haploidentical HCT) and high risk of GVHD. A second possible implication of CIKs' residual alloreactivity is its exploitation to ameliorate their ex-vivo expansion. A little modification in the standard CIKs' culture conditions, with the transient addition of allogeneic irradiated PBMC as stimulators, was shown to significantly increase the final expansion rate and augment the percentage of CD3+CD56+ cells without 
negatively affecting the antitumor activity or increasing their alloreactivity versus third party [16].

In general these findings are in favor of a possible use of CIK cells as alternative Donor Lymphocyte Infusion (DLI) following allogeneic HCT, with the possibility of further decreasing the GVHD in high-risk settings by depleting the CD3+CD56- fraction before the infusion. A second-generation DLI with CIK cells might increase the overall antitumor activity of HCT and improve its safety profile. Such positive evolution might help new experimental perspectives of HCT like those exploring its application as immunotherapy treatment for solid tumors.

\section{Tumor killing ability of CIK cells}

The ability to efficiently kill tumor cells is the ultimate basic ability requested to immune effectors candidate for adoptive immunotherapy. CIK cells are endowed with a MHC-independent tumor killing capacity against both solid and hematologic malignancies. The antitumor activity is mainly, even if not exclusively, associated with the CD3+CD56+ fraction and it is not due to the highest percentage of CD8+ cells compared to the CD3+CD56- subset[13]. The exact mechanism involved in tumor recognition and killing is not completely known but a main role seems to be played by the NKG2D molecule expressed on the membrane of CIK cells that interacts with MHC-unrestricted ligands on tumor cells [22]. The main targets recognized by NKG2D are MIC A/B (MHC class I related molecules) and proteins of the ULBP family (ULBPs 1,2 and 3) [23-25] while the final killing is perforin and granzyme mediated. NKG2D has a co-stimulatory role on conventional $\mathrm{T}$ lymphocytes, while on CIK cells it acquires a tumor-receptor function, following the ex-vivo activation with IL2 and upregulation of the DAP10 adaptor molecule [22]. MIC A/B are stress-induced proteins expressed by a wide range of malignant or transformed cells, their expression is MHC-unrestricted and not limited to a specific hystotype [26]. The expression of such ligands may also vary within the same tumor type, according to variations in the biological behavior and aggressiveness of tumor phenotype. We recently reported, in a breast cancer preclinical model, that acquisition of resistance to treatment with Trastuzumab could associate with the increased expression of MIC A/B on tumor cells and consequent augmented susceptibility to immune-mediated killing by CIK cells [27]. The antitumor activity of CIK cells have been described in vitro against several hematologic and solid malignancies including lung, gastrointestinal and mesenchymal tumors [13, 28-31]. The tumor killing activity was confirmed in vivo with murine models of tumor xenograft [32-34]. CIK cells are usually infused intravenously in the animal, they were shown to efficiently infiltrate tumor sites and demonstrated a superior activity compared to classic LAK cells against Non Hodgkin Lymphoma [2, 3, 35]. The majority of preclinical data were produced using allogeneic tumor cell lines as targets to demonstrate the efficacy of CIK cells against solid tumors. While cell lines may represent a useful tool to generate important proof of concepts, they may not fully account for the unique patient-specific biological and immunogenic characteristics of autologous tumor cells. Furthermore results may be influenced, at least in part, by HLA-mismatches between CIK cells and tumor targets. It is of great importance that future experiments may consider a design involving autologous tumor settings, to generate reliable patient-specific data that could serve as basis for subsequent clinical translation. In this direction first reports came by the Stanford group that showed activity of CIK cells against primary human ovarian cancer [33] and our group that recently reported data of intense tumor killing by patient-derived CIKs against autologous bone sarcoma cells [36]. An intriguing perspective for adoptive immunotherapy strategies with CIK cells is the emerging possibility to redirect and potentiate their antitumor activity with TAA bispecific antibodies [37] or their engineering to produce specific cytokines enhancing the cytotoxicity [38] or with tumor-receptor molecules [39] [40, 41].

\section{Clinical Studies}

The clinical translation of adoptive immunotherapy with CIK cells as treatment for patients with solid tumors is currently the object of clinical trials and their number has increased in the very recent years. The first clinical experience included 10 patients with metastatic renal carcinoma, colorectal cancer and lymphoma. Circulating CIK cells persisted for up to 2 weeks after the infusion, no relevant associated toxicities were reported. One patient with lymphoma obtained a complete response while six patients had progressive diseases and three did not experience any change. In this first study, CIK cells were engineered to auto-generate IL-2 [38]. Other clinical trials subsequently confirmed the safety and feasibility of this immunotherapy approach along with demonstration of initial clinical activity. In a phase I study reported on 12 patients with advanced non-Hodgkin's lymphoma (NHL), metastatic renal cancer or hepatocellular carcinoma (HCC), the adoptive infusion of CIK cells resulted in three complete responses and two stabilizations of disease. Two of the complete responses were observed in metastatic 
renal cancer and HCC, these responding patients received the simultaneous subcutaneous injection of low dose IL-2 and IFN-a respectively [42]. The above mentioned are, to our knowledge, the only two trials with CIK cells for solid tumors that have currently been published in Europe or U.S. A higher number of clinical studies have been recently performed in Asia with very interesting clinical results. In the setting of hepatocellular carcinoma, adjuvant infusions of autologous CIK cells after surgical resection, showed a significant increase in disease-free survival [43, 44]; an interesting observation was the reduction of $\mathrm{HBV}$ viral load associated with CIK treatments [45]. The adoptive infusion of CIK cells produced interesting clinical responses in patients with lung and gastric cancers with a reported positive impact on survival in association with chemotherapy [46-49]. A summary of clinical trials with CIK cells for the treatment of solid tumors is reported in table 1.

Overall these studies confirmed the high safety of adoptive immunotherapy with CIK cells with important indications suggestive for a clinical activity; the heterogeneity in methods and criteria for response assessment make still early and difficult to draw definitive statements concerning tumor response or impact on survival. Recently it has been created an
International Registry on CIK Cells (IRCC) with the aim of collecting data worldwide and setting standard criteria to report results from clinical trial with CIK cells [50]. Similar initiatives are very important as they could help a rational development of clinical research in the field and provide useful tools to analyze past and future results. These issues are crucial in order to derive objective and reliable conclusions on the efficacy of CIK cells as adoptive immunotherapy for cancer.

\section{Conclusions}

CIK cells represent a promising tool in the scenario of cancer adoptive immunotherapy strategies. Their easy and inexpensive ex-vivo expansion, along with the MHC-unrestricted tumor killing ability may overcome some crucial problems that have limited the diffusion and clinical translation of other immunotherapy approaches. The reduced alloreactivity across major HLA-barriers may open new perspective applications of CIK cells as alternative to conventional DLI after HCT, helping the extension of HCT to other experimental settings like the treatment of solid tumors.

Table 1. Clinical trials of adoptive immunotherapy with CIK cells as treatment for solid tumors.

\begin{tabular}{|c|c|c|c|c|c|}
\hline Disease & Patients & Type of CIKs & Toxicity & Clinical Responses & Ref. \\
\hline $\begin{array}{l}\text { Colorectal and renal carci- } \\
\text { noma; NHL }\end{array}$ & 10 & Autologous & Fever: 3 & CR (1) SD (3) & [38] \\
\hline $\begin{array}{l}\text { NHL; Renal Carcinoma.; } \\
\text { HCC }\end{array}$ & 12 & Autologous & Fever: 2 & $\mathrm{CR}(3) ; \mathrm{SD}(2)$ & [42] \\
\hline Advanced NSCLC & 59 (randomized) & $\begin{array}{l}\text { Autologous } \\
\text { (+ Chemotherapy) }\end{array}$ & None relevant & Improved PFS and OS & [46] \\
\hline $\begin{array}{l}\text { Resected HCC } \\
\text { (adjuvant setting) }\end{array}$ & 127 (randomized) & Autologous & Fever: 5 & Improved DFS & [43] \\
\hline HCC (adjuvant setting) & 85 (randomized) & Autologous & None relevant & Reduced recurrence rate & [44] \\
\hline Gastric cancer (Stage IV) & 57 (randomized) & $\begin{array}{l}\text { Autologous } \\
(+ \text { chemotherapy) }\end{array}$ & None Relevant & $\begin{array}{l}\text { Reduced Tumor Markers; Improved } \\
\text { QOL }\end{array}$ & [47] \\
\hline $\mathrm{HCC}$ & 13 & Autologous & $\begin{array}{l}\text { Transient Fever } \\
\text { (most of pts) }\end{array}$ & $\begin{array}{l}\text { Reduced tumor volume (3); Im- } \\
\text { proved symptoms; Decreased } \\
\text { HBV-DNA load }\end{array}$ & [45] \\
\hline $\begin{array}{l}\text { Various (NLSLC; gastroin- } \\
\text { testinal) }\end{array}$ & 40 (randomized) & $\begin{array}{l}\text { Allogeneic } \\
\text { (CB-derived) }\end{array}$ & None Relevant & Improved PFS and OS & [49] \\
\hline Gastric Cancer & 156 (randomized) & $\begin{array}{l}\text { Autologous }(+ \\
\text { Chemotherapy) }\end{array}$ & None Relevant & Improved Survival & [48] \\
\hline
\end{tabular}




\section{Conflict of Interest}

The author has declared that no conflict of interest exists.

\section{References}

1. Schmidt-Wolf I.G, et al. Phenotypic characterization and identification of effector cells involved in tumor cell recognition of cytokine-induced killer cells. Exp Hematol, 1993. 21(13): 1673-9.

2. Schmidt-Wolf I.G, et al. Use of a SCID mouse/human lymphoma model to evaluate cytokine-induced killer cells with potent antitumor cell activity. J.Exp.Med, 1991. 174(1): 139-149.

3. Lu P.H and Negrin R.S. A novel population of expanded human CD3+CD56+ cells derived from T cells with potent in vivo antitumor activity in mice with severe combined immunodeficiency. J. Immunol, 1994. 153(4): 1687-1696.

4. Introna $\mathrm{M}$, et al. Rapid and massive expansion of cord blood-derived cytokine-induced killer cells: an innovative proposal for the treatment of leukemia relapse after cord blood transplantation. Bone Marrow Transplant, 2006. 38(9): 621-627.

5. Hayes M.P, Wang J, and Norcross M.A. Regulation of interleukin-12 expression in human monocytes: selective priming by interferon-gamma of lipopolysaccharide-inducible p35 and p40 genes. Blood, 1995. 86(2): 646-50.

6. Ma X, et al. The interleukin $12 \mathrm{p} 40$ gene promoter is primed by interferon gamma in monocytic cells. J Exp Med, 1996. 183(1): 147-57.

7. Lopez R.D, et al. CD58/LFA-3 and IL-12 provided by activated monocytes are critical in the in vitro expansion of CD56+ T cells. Cancer Immunol Immunother, 2001. 49(12): 629-40.

8. Ochoa A.C, et al. Long-term growth of lymphokine-activated killer (LAK) cells: role of anti-CD3, beta-IL 1, interferon-gamma and -beta. Immunol J, 1987. 138(8): 2728-33.

9. Anderson P.M, Bach F.H, and Ochoa A.C. Augmentation of cell number and LAK activity in peripheral blood mononuclear cells activated with anti-CD3 and interleukin-2. Preliminary results in children with acute lymphocytic leukemia and neuroblastoma. Cancer Immunol Immunother, 1988. 27(1): 82-8.

10. Zoll B, et al. Generation of cytokine-induced killer cells using exogenous interleukin-2, -7 or -12 . Cancer Immunol Immunother, 1998. 47(4): 221-6.

11. Leemhuis $\mathrm{T}$, et al. A phase I trial of autologous cytokine-induced killer cells for the treatment of relapsed Hodgkin disease and non-Hodgkin lymphoma. Biol Blood Marrow Transplant, 2005. 11(3): 181-7.

12. Baker J, et al. Expansion of cytolytic CD8(+) natural killer T cells with limited capacity for graft-versus-host disease induction due to interferon gamma production. Blood, 2001. 97(10): 2923-31.

13. Sangiolo D, et al. Alloreactivity and anti-tumor activity segregate within two distinct subsets of cytokine-induced killer (CIK) cells: implications for their infusion across major HLA barriers. Int Immunol, 2008. 20(7): 841-8.

14. Bonanno G, et al. Thymoglobulin, interferon-gamma and interleukin-2 efficiently expand cytokine-induced killer (CIK) cells in clinical-grade cultures. J Transl Med, 2010. 8: 129.

15. $\mathrm{Li} \mathrm{H}$, et al. CD4 +CD25 + regulatory T cells decreased the antitumor activity of cytokine-induced killer (CIK) cells of lung cancer patients. J Clin Immunol, 2007. 27(3): 317-26.

16. Todorovic M, Gammaitoni L, Leuci V, et al. Ex-vivo Allogeneic Stimulation Significantly Improves Expansion of Cytokine-Induced Killer Cells without Increasing their Alloreactivity across MHC-barriers. EHA. 2011; epub.

17. Linn Y.C, et al. Characterization of the recognition and functional heterogeneity exhibited by cytokine-induced killer cell subsets against acute myeloid leukaemia target cell. Immunology, 2009 Mar;126(3):423-35.

18. Verneris M.R, et al. Engineering hematopoietic grafts: purified allogeneic hematopoietic stem cells plus expanded CD8+ NK-T cells in the treatment of lymphoma. Biol.Blood Marrow Transplant, 2001. 7(10): 532-542.

19. Yang Y.G, et al. Donor-derived interferon gamma is required for inhibition of acute graft-versus-host disease by interleukin 12. J Clin Invest, 1998. 102(12): 2126-35.

20. Nishimura $R$, et al. In vivo trafficking and survival of cytokine-induced killer cells resulting in minimal GVHD with retention of antitumor activity. Blood, 2008. 112(6): 2563-74.

21. Introna $\mathrm{M}$, et al. Repeated infusions of donor-derived cytokine-induced killer cells in patients relapsing after allogeneic stem cell transplantation: a phase I study. Haematologica, 2007. 92(7): 952-959.

22. Verneris M.R, et al. Role of NKG2D signaling in the cytotoxicity of activated and expanded CD8+ T cells. Blood, 2004. 103(8): 3065-3072.

23. Jamieson A.M, et al. The role of the NKG2D immunoreceptor in immune cell activation and natural killing. Immunity, 2002. 17(1): 19-29.

24. Diefenbach A, et al. Ligands for the murine NKG2D receptor: expression by tumor cells and activation of cells NK and macrophages. Nat Immunol, 2000. 1(2): 119-26.

25. Cosman D, et al. ULBPs, novel MHC class I-related molecules, bind to CMV glycoprotein UL16 and stimulate NK cytotoxicity through the NKG2D receptor. Immunity, 2001. 14(2): 123-33.

26. Groh V, et al. Broad tumor-associated expression and recognition by tumor-derived gamma delta T cells of MICA and MICB. Proc Natl Acad Sci U S A, 1999. 96(12): 6879-84.

27. Sangiolo D, et al. MIC A/B Protein Expression Associates with Trastuzumab-Resistant Breast Cancer Cells Leading to Effective Immunotherapy by Cytokine Induced Killer Cells. ASH. 2009; epub.

28. Schmidt-Wolf I.G, et al. Sensitivity of multidrug-resistant tumor cell lines to immunologic effector cells. Cell Immunol, 1996. 169(1): 85-90.

29. Sangiolo D, et al. Cytokine induced killer cells as adoptive immunotherapy strategy to augment graft versus tumor after hematopoietic cell transplantation. Expert Opin Biol Ther, 2009. 9(7): 831-40.

30. Kuci S, et al. Efficient lysis of rhabdomyosarcoma cells by cytokine-induced killer cells: implications for adoptive immunotherapy after allogeneic stem cell transplantation. Haematologica, 2010. 95(9): 1579-86.

31. Hongeng S, et al. Generation of CD3+ CD56+ cytokine-induced killer cells and their in vitro cytotoxicity against pediatric cancer cells. Int Hematol J, 2003. 77(2): 175-9.

32. Wongkajornsilp A, et al. Human cytokine-induced killer cells specifically infiltrated and retarded the growth of the inoculated human cholangiocarcinoma cells in SCID mice. Cancer Invest, 2009. 27(2): 140-8.

33. Chan J.K, et al. Enhanced killing of primary ovarian cancer by retargeting autologous cytokine-induced killer cells with bispecific antibodies: a preclinical study. Clin.Cancer Res, 2006. 12(6): 1859-1867.

34. Kim H.M, et al. Antitumor activity of cytokine-induced killer cells in nude mouse xenograft model. Arch Pharm Res, 2009. 32(5): 781-7.

35. Schmidt-Wolf I.G, et al. Propagation of large numbers of T cells with natural killer cell markers. Br.J.Haematol, 1994. 87(3): 453-458.

36. Sangiolo D, et al. Effective preclinical adoptive immunotherapy with cytokine-induced killer cells for bone sarcomas. EBMT. 2011; epub. 
37. Verneris M.R, et al. Low levels of Her2/neu expressed by Ewing's family tumor cell lines can redirect cytokine-induced killer cells. Clin Cancer Res, 2005. 11(12): 4561-70.

38. Schmidt-Wolf I.G, et al. Phase I clinical study applying autologous immunological effector cells transfected with the interleukin-2 gene in patients with metastatic renal cancer, colorectal cancer and lymphoma. Br Cancer J, 1999. 81(6): 1009-16.

39. Tita-Nwa F, et al. Cytokine-induced killer cells targeted by the novel bispecific antibody CD19xCD5 (HD37xT5.16) efficiently lyse B-lymphoma cells. Cancer Immunol Immunother, 2007. 56(12): 1911-20.

40. Marin V, et al. Enhancement of the anti-leukemic activity of cytokine induced killer cells with an anti-CD19 chimeric receptor delivering a 4-1BB-zeta activating signal. Exp Hematol, 2007. 35(9): 1388-97.

41. Yoon S.H, et al. Transfer of Her-2/neu specificity into cytokine-induced killer (CIK) cells with RNA encoding chimeric immune receptor (CIR). J Clin Immunol, 2009. 29(6): 806-14.

42. Olioso $\mathrm{P}$, et al. Immunotherapy with cytokine induced killer cells in solid and hematopoietic tumours: a pilot clinical trial. Hematol Oncol, 2009; epub.

43. Hui D, et al. A randomized controlled trial of postoperative adjuvant cytokine-induced killer cells immunotherapy after radical resection of hepatocellular carcinoma. Dig Liver Dis, 2009. 41(1): 36-41.

44. Weng D.S, et al. Minimally invasive treatment combined with cytokine-induced killer cells therapy lower the short-term recurrence rates of hepatocellular carcinomas. Immunother J, 2008. 31(1): 63-71.

45. Shi M, et al. Autologous cytokine-induced killer cell therapy in clinical trial phase I is safe in patients with primary hepatocellular carcinoma. World Gastroenterol J, 2004. 10(8): 1146-51.

46. Wu C, et al. Prospective study of chemotherapy in combination with cytokine-induced killer cells in patients suffering from advanced non-small cell lung cancer. Anticancer Res, 2008. 28(6B): 3997-4002.

47. Jiang J, et al. Treatment of advanced gastric cancer by chemotherapy combined with autologous cytokine-induced killer cells. Anticancer Res, 2006. 26(3B): 2237-2242.

48. Jiang J.T, et al. Increasing the frequency of CIK cells adoptive immunotherapy may decrease risk of death in gastric cancer patients. World Gastroenterol J, 2010. 16(48): 6155-62.

49. Niu Q, et al. Cord blood-derived cytokine-induced killer cells biotherapy combined with second-line chemotherapy in the treatment of advanced solid malignancies. Int Immunopharmacol, 2011. 11(4): 449-56.

50. Hontscha C, et al. Clinical trials on CIK cells: first report of the international registry on CIK cells (IRCC). J Cancer Res Clin Oncol, 2011. 137(2): 305-10. 